\title{
CALIBRATING AND TESTING NEW ULTRACAM SENSORS
}

\author{
Michael Gruber, Alexander Wiechert \\ Vexcel Imaging GmbH8010 Graz, Austria \\ \{michael.gruber, alexander.wiechert\}@vexcel-imaging.com
}

EuroCOW 2017

KEY WORDS: Digital photogrammetry, digital workflow, digital aerial camera, aerial sensor \begin{abstract}
This contribution reports on the latest design of new UltraCam sensors. We present results from calibration and verification flights of the UltraCam Condor, which is based on a specific design and offers highest productivity from its 38000 pixel cross track image format. UltraCam Condor is based on the successful project experience with its predecessor, the UltraCam G.
\end{abstract}

\section{INTRODUCTION}

UltraCam digital camera systems stand for high quality and are optimized for acquiring digital aerial images as well as images taken from our terrestrial sensors from ground level. Since the introduction of UltraCam D in 2003 Vexcel Imaging has continuously developed the product portfolio of large format aerial sensors as well as terrestrial sensors and the software to operate the sensors, process images and cover the entire digital photogrammetric workflow (Wiechert et al., 2013).

During the years with Microsoft from 2006 to 2016 Vexcel Imaging contributed to large photogrammetric projects like the Global Ortho project in cooperation with Digital Globe (Walcher et al., 2012). This huge photogrammetric project was based on the UltraCam $\mathrm{G}$ digital aerial sensor with a swath width of 28000 pixel. The success of UltraCam $\mathrm{G}$ encouraged us to develop a successor, the UltraCam Condor. This 38000 pixel camera is larger and thus more productive than the UltraCam $\mathrm{G}$ and is equipped with up to date components like solid state storage units and the state of the art electronic boards we developed for our UltraCam sensor products.

\section{THE ULTRACAM SENSOR FAMILY}

Vexcel Imaging Graz operates as an independent company since March 2016 and thus we developed a new and modern arrangement for our products. The aerial sensors are optimized for three specific project scenarios, namely the high-performance nadir photogrammetric cameras UltraCam Eagle and UltraCam Falcon, the Nadir and Oblique sensor UltraCam Osprey (Wiechert et al., 2017) and the wide area mapping sensor UltraCam Condor. Figure 1 shows the family of UltraCam aerial and terrestrial sensors.
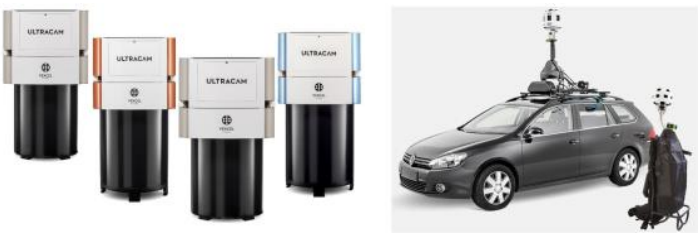

Fig. 1: UltraCam Aerial Sensor Family: UltraCamFalcon, UltraCam Condor, UltraCam Eagle and UltraCam Osprey (from left to right). With the Eagle we offer 4 different lens packages, the Osprey is available in two different versions. UltraCam Mustang and UltraCam Panther (on the right) are designed for terrestrial data acquisition.

Beside our aerial camera products for efficient aerial data acquisition we do have terrestrial sensors in our portfolio. The UltraCam Mustang is our street-side system which was developed to contribute to the Bing Maps data acquisition and is now available as a sensor product for a huge variety of applications. The UltraCam Panther, our portable system for all off road and indoor mapping projects is now under development and will be available by end of the calendar year (cf. Fig. 1).

The UltraMap software product is Vexcel's software package which offers all modules to manage raw UltraCam image data, process

UltraCam Images and introduce these data into the photogrammetric production chain. The photogrammetric processing chain UltraMap was updated and offers all capabilities to handle all new Vexcel cameras like UltraCam Condor.
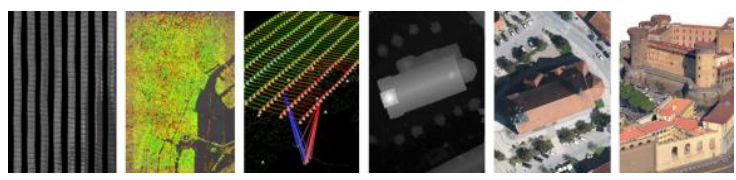

Fig. 2: UltraMap offers the processing chain for UltraCam images. Results are image orientation (AT), interactive and automated measurements, surface models, ortho images and three-dimensional object models. 


\section{ULTRACAM CONDOR}

UltraCam Condor and its predecessor UltraCam G were developed for mapping very large areas. If such large project shall be acquired by aerial operations a powerful and efficient sensor system needs to be in place. UltraCam Condor is the answer to enable such large photogrammetric activities. It is the most recent offering in Vexcel Imaging's suite of high-performance UltraCam digital aerial systems.

UltraCam Condor addresses a very specific application: high altitude ortho image generation with exceptional image quality. Producing a camera system that meets all the requirements of high altitude ortho production presented several complex challenges in the design of the lens system, the electronics and the storage system, as well as significant investment in the UltraMap processing software to handle necessary radiometric corrections and eliminate artifacts. The UltraCam Condor is positioned to redefine the efficiency/quality ratio in large area/nationwide collection efforts and transform user expectations for project timelines and refresh cycles.

\begin{tabular}{|l|l|l|}
\hline $\begin{array}{l}\text { Color (RGB Bayer } \\
\text { pattern) }\end{array}$ & $38,000 \times 5,000$ pixels & Pixel Size \\
\hline PAN & $13,400 \times 8,650$ pixels & $5.2 \mu \mathrm{m}$ \\
\hline $\begin{array}{l}\text { Color (NIR) } \\
\begin{array}{l}\text { Color capability } \\
\text { (multi-spectral) }\end{array}\end{array}$ & $4,600 \times 5,000$ pixels & $4.6 \mu \mathrm{m}$ \\
\hline $\begin{array}{l}\text { Ratio RGB to PAN } \\
\text { to NIR }\end{array}$ & $1: 2.83: 4.35$ \\
\hline Frame rate & 1 frame per 1.75 seconds & \\
\hline Weight & $64 \mathrm{~kg}$ & \\
\hline Power consumption & Max. $350 \mathrm{~W}$ & $\begin{array}{l}\text { Lens } \\
\text { aperture }\end{array}$ \\
\hline $\begin{array}{l}\text { Color (RGB Bayer } \\
\text { pattern) }\end{array}$ & $100 \mathrm{~mm}$ & $\mathrm{f}=1 / 5.6$ \\
\hline PAN & $40 \mathrm{~mm}$ & $\mathrm{~F}=1 / 4.8$ \\
\hline $\begin{array}{l}\text { Color (NIR) } \\
\text { Flying height for } \\
\text { RGB @ 10 cm GSD }\end{array}$ & $23 \mathrm{~mm}$ & $\mathrm{~F}=1 / 5.6$ \\
\hline
\end{tabular}

Table 1: Key parameters of UltraCam Condor

\section{DESIGN PRINCIPALS OF ULTRACAM CONDOR}

The UltraCam Condor is a multi-cone camera system. We use multiple detector arrays (CCD sensor arrays) and multiple optical systems of different optical length to build one large format camera system.

The photogrammetric backbone of the UltraCam Condor is the $13 \mathrm{k}$ by $9 \mathrm{k}$ panchromatic nadir camera, which consist of two camera heads and two medium size CCD sensor arrays. The focal length of this camera is $40 \mathrm{~mm}$ and thus a physical image scale of 1: 100000 is derived at a flying altitude of $4000 \mathrm{~m}$ above ground level. This leads to a ground sampling distance of $52 \mathrm{~cm}$ at a pixel size of 5,2 $\mu \mathrm{m}$.

The color cones are equipped with $100 \mathrm{~mm}$ optical lens systems and thus the physical image scale is $1: 40000$. Due to the smaller pixel size of the color CCD sensor arrays of $4,6 \mu \mathrm{m}$ one derives a ground sampling distance of $18,4 \mathrm{~cm}$ from $4000 \mathrm{~m}$ flight altitude.

The set of color images build together a rectangular footprint on the ground with a very large cross track dimension of 38000 pixels and a smaller long track dimension of 5000 pixels. During the postprocessing all color sub-images from four separate camera heads are stitched together and are co-registered onto the panchromatic image. An additional camera head is used to capture NIR images as well at a focal length of $23 \mathrm{~mm}$ and the NIR channel is co-registered onto the panchromatic images as well.

The flight mission layout can be tuned to the desired resolution of the color image and the endlap of RGB can be set to $20 \%$ only. This is enough for a gapless coverage on the ground. The corresponding endlap for the panchromatic image is then at $83 \%$, which is far enough for a strong aero-triangulation set up.
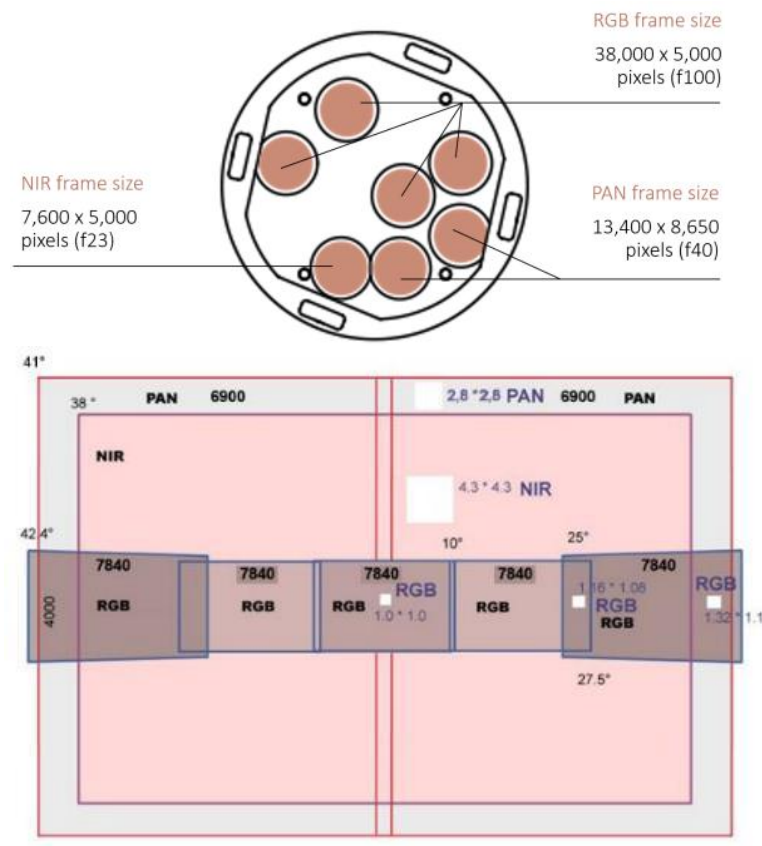

Fig 3: Cone layout of the UltraCam Condor (top) and footprint extend for PAN, RGB and NIR (bottom).

A short overview of benefits of such design is given below:

the use of multiple lens systems avoids the use of one expensive large format lens system;

the camera can be operated within a standard environment (standard mount and single hole aircraft);

the image of the panchromatic camera sub system is enhanced by the large format color image of the RGB camera sub system in such way that parts of the footprint of the panchromatic system are superimposed by the higher resolution RGB image to improve manual measurements;

The panchromatic backbone further enables automated dense matching, DSM/DTM and ortho image generation. That makes additional flights by other sensors obsolete as all necessary data sets cam be derived from one UltraCam condor flight;

the determination of the exterior orientation of the system based on standard AAT making use 
of the overlapping images from the panchromatic sub camera;

The panchromatic and the RGB camera systems are triggered in such way, that the exposures of the multiple camera systems are taken at one and the same position exploiting the known speed of the aircraft over ground (syntopic exposure).

The image extent and the full resolution of the high resolution RGB color image is illustrated below. Figure 4 shows the 38000 by 5000 pixel and a crop of 1300 by 1000 pixel. The RGB ground sampling distance (GSD) of this frame is at $10 \mathrm{~cm}$.

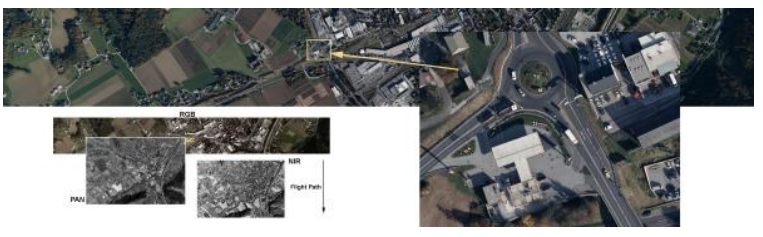

Fig. 4: Results of an UltraCam Condor photo mission at $2200 \mathrm{~m}$ AGL and $10 \mathrm{~cm}$ GSD. The crop area on the right shows a $130 \mathrm{~m}$ by $100 \mathrm{~m}$ portion of the RGB image. The

footprint for PAN, RGB and NIR is illustrated at the lower left part of this figure.

\section{CALIBRATION OF THE ULTRACAM CONDOR}

The process of the laboratory calibration of UltraCam Condor is the initial step to estimate all parameters of the sensor head. In this contribution we focus on the geometric calibration and present the set up of this wide angle system (cf. Figure 5 a).

During the data capture process of the calibration we register the temperature at each lens cone of the seven independent camera heads and the temperature at each of the eight CCD sensor packages. Due to a sufficient warm up phase these temperature readings are quite constant during the data capture process. The average of each set of these readings can be stored as one result of the lab calibration (cf. Figure 5b). These temperature values are then used during the stitching process of each production image and to compute the temperature differences between calibration and production flight.
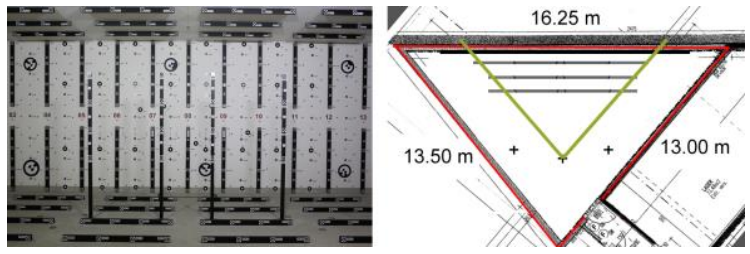

Fig. 5 a: Calibration structure (left) and floorplan with three camera positions (+) and FOV of the UltraCam Condor (green triangle).

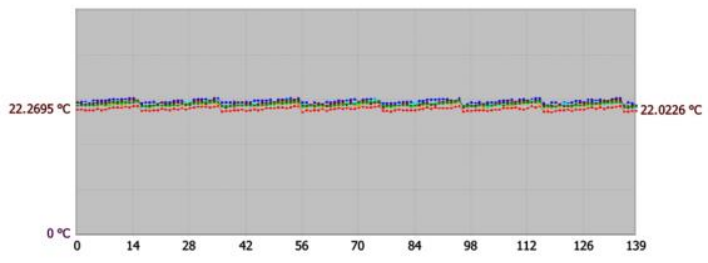

Fig. 5 b: Measured lens temperatures during the calibration set-up. The temperature range of each lens for the entire set is at $1,2^{\circ} \mathrm{C}$. The standard deviation of the set of 140 measurements for each individual lens system is in the range of $0,3^{\circ} \mathrm{C}$. The mean temperature value from each lens is stored and reused for the postprocessing of each individual production image.

Important results from the calibration are measured coordinates of the target point in a set of images taken during the calibration procedure. The standard layout of the calibration consists of 84 photos taken from the 3 positions at 4 tilt angles $\left(0^{\circ}, 90^{\circ}, 180^{\circ}\right.$ and $\left.270^{\circ}\right)$ and 7 rotations. In order to improve the results for the special frame format of the UltraCam Condor RGB camera we have introduced 56 additional photos at a $30^{\circ}, 60^{\circ}, 120^{\circ}$, $150^{\circ}, 210^{\circ} 240^{\circ}, 300^{\circ}$ and $330^{\circ}$ at the central camera station. The set of observations are then introduced into the Bingo bundle adjustment software and a least squares solution for the set up is calculated (see also Gruber et al., 2016).
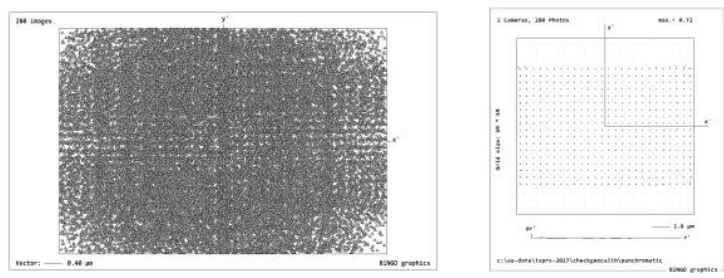

Fig. 6 a: Result from the Panchromatic Camera. The position of all measured target points in the image plane is illustrated on the left. The remaining image residuals after the calibration is shown on the right.

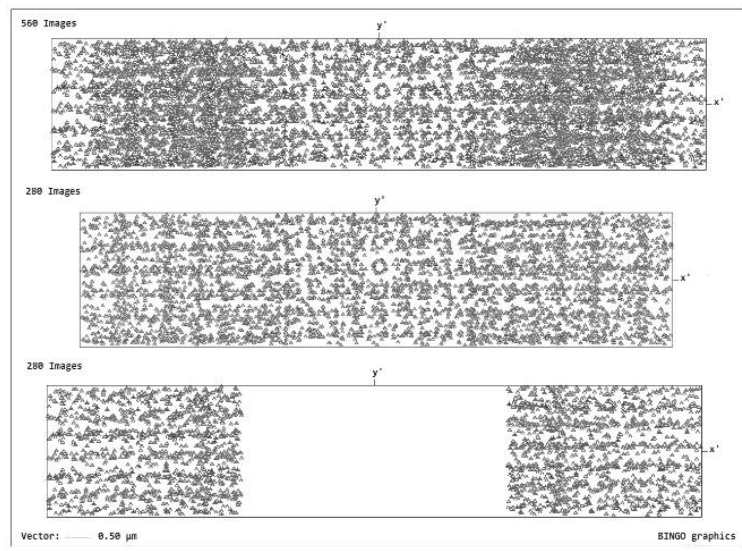

Fig. 6b : Result from the RGB Color Camera. All camera heads are on top and the slightly tilted camera heads on the left and right area of the image extend are on the bottom. The two cones from the center part of the frameare shown in the middle of the figure. 
The primary input data for the bundle adjustment and the computation of all camera parameters are the measured image positions of the calibration targets. For the panchromatic camera heads (two cones) a number 35000 image positions from 280 photos were automatically detected. For the RGB color component the number of measured point positions was 9200 and for the NIR camera head 30000 points. The overall quality of the adjustment is reported from the sigma_o value, which was in the range of 0,5 at an a priori standard deviation of image coordinate measurements of $2 \mu \mathrm{m}$. The a posteriori RMS error value for image coordinate measurements of the panchromatic camera was $0,4 \mu \mathrm{m}$ for $\mathrm{x}$ and $\mathrm{y}$.

\section{RESULTS FROM THE VERIFICATION FLIGHT}

The verification flight in a region near Graz illustrates the potential of UltraCam Condor. The project was flown at an altitude of $2200 \mathrm{~m}$ above mean ground level and consists of 126 photos from 7 flight-lines, 4 lines N-S, 3 lines E-W. The results from the automated aero-triangulation by UltraMap AT show very good numbers from the panchromatic camera at the GSD of $28 \mathrm{~cm}$. The sigma_o value of 0.77 corresponds with the RMS error of image measurements of $0.7 \mu \mathrm{m}$ in $\mathrm{x}$ and $\mathrm{y}$. The RMS errors of check points and GCPs were below the $5 \mathrm{~cm}$ level for X, $\mathrm{Y}$ and $\mathrm{Z}$.

Results from the block set up are shown in Figure 7.
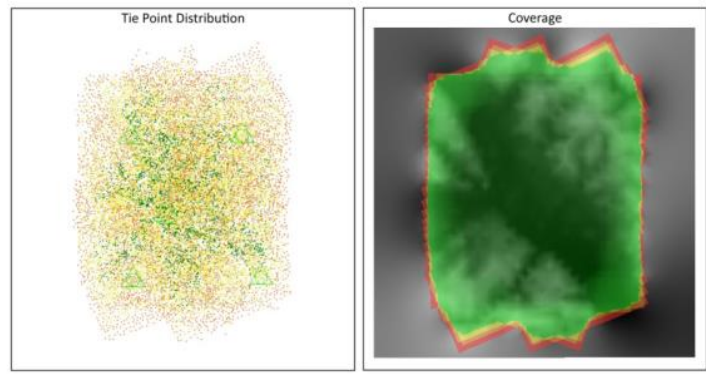

Fig. 7: Results from an UltraCam Condor flight mission from the UltraMap AT-report: The block layout on the left shows the distribution of automatically measured tie points. The coverage on the ground is illustrated on the right. Multiple overlays are color coded.

\section{CONCLUSION}

In this contribution, we explained the new design of the sensor product portfolio from Vexcel Imaging since the separation from Microsoft in 2016. As an aerial camera system with some specific design features we focused on the UltraCam Condor and illustrated details from the calibration procedure and a flight mission.

\section{REFERENCES}

Wiechert, A., Gruber, M., 2017: News from UltraCam Sensors - an Update, Proceedings of the American Society for Photogrammetry \& Remote Sensing, 12-16 March 2017, Baltimore, MD.

Gruber, M., B. Schachinger, M. Muick, Ch. Neuner, H. Tschemmernegg, 2016: Geometric Calibration and Validation of UltraCam Aerial Sensors, EuroCOW 2016, 10-1 February 2016, Lausanne, CH.

Wiechert, A., Gruber, M., 2013: News from the UltraCam camera line-up, Proceedings of the American Society for Photogrammetry \& Remote Sensing, 25-29 March 2013, Baltimore, MD.

Walcher W., Leberl F., Gruber M., 2012: The Microsoft Global Ortho Program, International Archives of the Photogrammetry, Remote Sensing and Spatial Information Sciences, Melbourne 2012, Australia. 